\title{
Evaluation of the efficacy of laparoscopic-assisted radical vaginal hysterectomy and abdominal radical hysterectomy for treating cervical cancer: a meta-analysis
}

\author{
Zhen Zeng, Jia Liu, Tao Lv, Zonghao Feng, Lei Zhang, Qinping Liao \\ Department of Obstetrics and Gynecology, Beijing Tsinghua Changgung Hospital, School of Clinical Medicine, Tsinghua University, \\ Beijing, China
}

Videosurgery Miniinv 2022; 17 (1): 69-82 DOI: https://doi.org/10.5114/wiitm.2021.106126

\begin{abstract}
Introduction: Laparoscopic-assisted radical vaginal hysterectomy (LARVH) and abdominal radical hysterectomy $(A R H)$ are commonly used for cervical cancer treatment. However, the clinical application of LARVH versus ARH in treating cervical cancer remains controversial.

Aim: To investigate the efficacy of $L A R V H$ versus $A R H$ in treating cervical cancer via comparing several inductors by pooling related studies.

Material and methods: Eligible articles from PubMed, Embase, and the Cochrane library were screened using established search terms. Consecutive variables were pooled using weighted mean difference (WMD) and 95\% confidence interval (CI). Categorical variables were pooled using odds ratio (OR) and $95 \% \mathrm{Cl}$.

Results: $A$ total of 13 articles were included in this meta-analysis, comprising 579 patients who underwent LARVH and 810 who underwent $A R H$. LARVH required a longer operation time (WMD = 50.97, 95\% CI: 38.34, 63.59, $p<$ $0.001)$ than ARH. However, compared to patients who underwent $A R H$, those who underwent LARVH had less bleeding volume $(W M D=-311.21,95 \% \mathrm{Cl}:-482.77,-139.64, p<0.001)$, required a shorter hospital stay $(W M D=-3.38$, $95 \% \mathrm{Cl}:-5.00,-1.76, p<0.001)$, and had a lower risk of urinary tract infection (OR $=0.34,95 \% \mathrm{Cl}: 0.13,0.89, p=$ 0.028). Additionally, patients who underwent $L A R V H$ showed a slightly lower recurrence rate $(O R=0.549,95 \% \mathrm{Cl}$ : $0.302,0.998, p=0.049)$ than patients who underwent $A R H$. However, subgroup analysis results were not in agreement with the pooled results and indicated an unstable outcome.
\end{abstract}

Conclusions: Owing to these reasons, LARVH has more application prospects than ARH in treating cervical cancer.

Key words: meta-analysis, cervical cancer, laparoscopic-assisted radical vaginal hysterectomy, abdominal radical hysterectomy, efficacy comparison.

\section{Introduction}

Cervical cancer is a common lethal gynaecological malignancy affecting women worldwide. In Asia, over 500,000 new cases are diagnosed annually, with a mortality rate of about 50\% [1-3]. Abdominal radical hysterectomy $(\mathrm{ARH})$, first reported in 1900, is widely accepted as the gold standard treatment for patients with early stage cervical cancer $[4,5]$. With improvements in laparoscopic techniques, in the late 1980s, laparoscopic-assisted radical vaginal hysterectomy (LARVH) was developed for treating cervical cancer $[6,7]$. Owing to the controversial clinical efficacies of LARVH and ARH, determining the better treatment option for cervical cancer is challenging $[8,9]$. 
Several studies have investigated the value of LARVH and ARH to determine the better choice for treating cervical cancer. All comparisons between LARVH and ARH for treating early stage cervical cancers mostly focused on blood loss, hospital stay, recovery, cosmetic results, and oncologic outcomes. However, the clinical application of LARVH versus ARH in treating cervical cancer remains controversial $[8,9]$. The application of laparoscopic procedures in oncologic surgery has many advantages, including short hospital stay and less blood loss during the preoperative period [10]. Studies reported that LARVH increased intraoperative complications, such as increased lymphocytes and nerve injury $[11,12]$. Recently, a study of meta-analysis investigated the efficacy of ARH and LARVH, and it suggested that attention should be paid to LARVH owing to its lower blood loss and shorter hospital stay [13]. However, significant heterogeneity was observed. Another study reported similar operative time between LARVH and ARH [14]. Thus, further studies that systematically assess the clinical efficacy of LARVH versus ARH are warranted.

\section{Aim}

Considering this, a meta-analysis was designed to compare the clinical efficacy of ARH versus LARVH in treating cervical cancer. Subgroup analyses were performed. From the results, we hope to provide some evidence for improving the outcome and prognosis of patients with cervical cancer.

\section{Material and methods}

\section{Search strategy}

Cervical cancer-associated studies published in English were searched from electronic databases, including PubMed, Embase, and the Cochrane Library. The retrieval period was up to 4 September 2020. Search terms were (LARVH OR (laparoscopic-assisted radical hysterectomy) OR (laparoscopic radical hysterectomy) OR (Laparoscopically Radical Hysterectomy)) AND (Cervical Cancer), and the search was performed by combining subject words with free words. To acquire more eligible studies, paper documents and references of relevant studies were manually searched.

The design was in accordance with PRISMA guidelines. For the research question, the PICO model was accepted.

\section{Selective criteria}

Studies involving adult women diagnosed with cervical cancer; those in which the efficacy of LARVH and $\mathrm{ARH}$ in treating cervical cancer was compared; those reporting one or more of the following outcomes: operative time, blood loss, hospital stay, or postoperative follow-up data such as recurrence rate, mortality, and complications; and those published in English were included.

Studies in which participants in experimental and control groups were family members or close relatives; reviews, comments, or letters; and duplicate publications were excluded. When the same population data were used for multiple studies, the study with more complete data was chosen.

\section{Data extraction and quality evaluation}

Studies were screened according to the above criteria. Subsequently, data such as basic information (first author's name, year of publication, study type, region and time period, cervical cancer stage, age, gender, and sample size of participants for experimental and control groups) and outcome data (relevant operative data, including operation time, blood loss, and length of stay; postoperative follow-up data, including recurrence rate, mortality, and complications) were retrieved. Additionally, data about FIGO stage, lymph node metastasis, the removed lymph nodes, adjuvant radiotherapy, rate of positive margins, team of operations, and surgery types were also retrieved.

The quality of included randomised control trials was evaluated using the Cochrane collaboration risk-of-bias tool and that of included cohort studies was assessed by the Newcastle-Ottawa Scale (NOS) [15]. All procedures were independently performed by two investigators; disagreements were resolved by discussion.

\section{Subgroup analysis}

Subgroup analyses were performed to explore the impacts of region and study type on outcomes of patients with cervical cancer in the two groups.

\section{Sensitivity analysis and publication bias}

Sensitivity analysis was performed using the 'leave one out' method to evaluate the stability of pooled data. Egger's test was applied to assess pub- 
lication bias. Sensitivity analysis and Egger's test were performed using Stata 13.0.

\section{Statistical analysis}

Continuous variables, such as operative time, blood loss, and length of hospital stay, were pooled using weighted mean difference (WMD) and 95\% confidence interval (Cl); categorical variables, such as recurrence rate, complications, and mortality, were pooled using odds ratio (OR) and $95 \% \mathrm{Cl}$. Cochran $\mathrm{Q}$ statistics and $l^{2}$ test were used to analyse heterogeneity among studies [16]. If significant heterogeneity was observed ( $p<0.05$ and/or $R^{2}>50 \%$ ), a random effects model was used for pooled analyses; otherwise, a fixed effects model was used. Data were analysed using Stata 13.0.

\section{Results}

\section{Study selection}

As shown in Figure 1, 1565 studies were selected, including 769 articles from PubMed, 691 from Embase, and 105 from the Cochrane Library. After reviewing the titles, 408 duplicate and 1131 unrelated articles were removed. Then, 26 articles were further reviewed and 13 were removed, including 5 single-arm studies on LARVH, 3 reviews, 3 meeting abstracts and 2 studies unrelated to LARVH. Finally, 13 articles were included [7, 14, 17-27].

\section{Characteristics of included studies}

Characteristics of included studies are listed in Table I. Only 1 of the 13 included articles was designed as a randomised controlled trial [22], 5 were prospective studies [17, 19, 22, 23, 26], and 8 were retrospective studies [7, 14, 18-20, 23, 24, 27]. Participants in the included studies came from different countries, including Korea, Bulgaria, China, the UK, Germany, Ireland, Spain, and Canada. All were diagnosed with cervical cancer; cancer stage was classified based on the criteria of the International Federation of Gynaecology and Obstetrics. In total, 1389 patients were included, among which 579 and 810 patients had undergone LARVH and ARH, respectively.

\section{Quality assessment}

Quality assessment revealed that 12 included studies, except for the study by Naik et al., had an NOS score of more than 4; in addition, most studies had an NOS score of more than 6 , suggesting that these studies had high quality (Table I). The quality of the study by Naik et al. was also high, with low risk for most evaluated biases (Figure 2).

\section{Meta-analysis of perioperative outcome}

In total, 11, 7, and 9 articles reported operation time, surgical bleeding, and length of hospital stay, respectively. Significant heterogeneity was observed among these studies $\left(p<0.05, l^{2}>50 \%\right)$; thus, pooled analyses of these data were performed using the random effects model.

Compared to ARH, LARVH required a longer operation time (WMD $=50.97,95 \% \mathrm{Cl}: 38.34,63.59, p<$ 0.001 ). However, compared to patients undergoing $\mathrm{ARH}$, those undergoing LARVH had lower bleeding volume (WMD $=-311.21,95 \% \mathrm{Cl}:-482.77,-139.64$, $p<0.001)$ and shorter hospital stay (WMD $=-3.38$, $95 \% \mathrm{Cl}:-5.00,-1.76, p<0.001$ ) (Figure 3).

Subgroup analyses of the study design (prospective vs. retrospective) and region (Asian vs. Western countries) were performed. All results of subgroup analyses were consistent with those of the overall analysis (Table II).

Egger's tests identified no significant publication bias in studies that reported regarding operation time $(p=0.620)$ and hospital stay $(p=0.297)$. However, there was significant publication bias among studies that reported on blood loss $(p=0.026)$. Moreover,

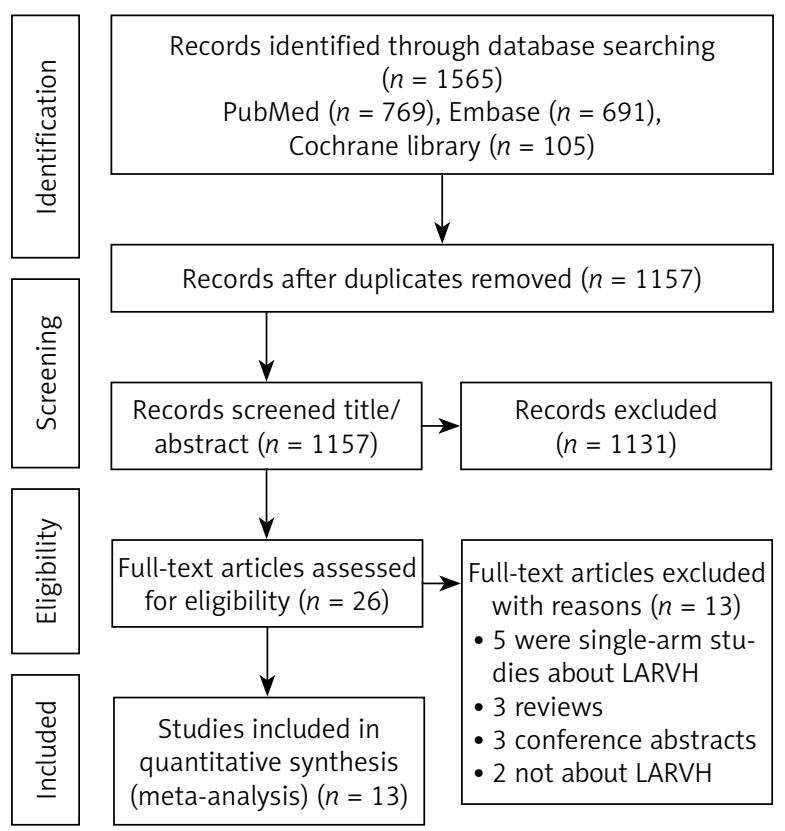

Figure 1. Flow diagram for article selection 
Table I. Characteristics of the included studies

\begin{tabular}{|c|c|c|c|c|c|c|c|c|c|}
\hline \multirow[t]{2}{*}{ Study } & \multirow{2}{*}{$\begin{array}{l}\text { Study } \\
\text { type }\end{array}$} & \multirow[t]{2}{*}{ Country } & \multirow{2}{*}{$\begin{array}{l}\text { Study } \\
\text { period }\end{array}$} & \multirow{2}{*}{$\begin{array}{c}\text { Type of } \\
\text { participants }\end{array}$} & \multicolumn{2}{|c|}{ No. of patients } & \multicolumn{2}{|c|}{ Age [years] } & \multirow{2}{*}{$\begin{array}{l}\text { Quality } \\
\text { assessment } \\
\text { (NOS) }\end{array}$} \\
\hline & & & & & LARVH & ARH & LARVH & ARH & \\
\hline Choi 2012 & $P$ & Korea & $\begin{array}{c}2004.09- \\
2010.12\end{array}$ & FIGO stage I-IIA & 89 & 99 & $\begin{array}{c}46 \\
(26-74)^{*}\end{array}$ & $\begin{array}{c}47 \\
(28-77)\end{array}$ & 7 \\
\hline $\begin{array}{l}\text { Gortchev } \\
2012\end{array}$ & $\mathrm{R}$ & Bulgaria & $\begin{array}{c}2006.01- \\
2010.05\end{array}$ & $\begin{array}{l}\text { T1B1 cervical } \\
\text { cancer }\end{array}$ & 46 & 175 & $42.5 \pm 9.9$ & $49.0 \pm 11.0$ & 6 \\
\hline Hou 2011 & $P$ & China & $\begin{array}{l}2009.05- \\
2010.07\end{array}$ & FIGO stage IA-IIB & 33 & 30 & $\begin{array}{c}47.55 \\
(27-63)\end{array}$ & $\begin{array}{c}44.94 \\
(27-79)\end{array}$ & 5 \\
\hline $\begin{array}{l}\text { Jackson } \\
2004\end{array}$ & $R$ & UK & 1993-2003 & $\begin{array}{l}\text { FIGO stage } \\
\text { 1A2-1B2 }\end{array}$ & 50 & 50 & $\begin{array}{c}45.7 \\
(27-81)\end{array}$ & $\begin{array}{c}45.5 \\
(24-86)\end{array}$ & 7 \\
\hline Malur 2001 & $\mathrm{R}$ & Germany & $\begin{array}{c}1991.01- \\
1994.03 \\
1994.08- \\
1999.05\end{array}$ & FIGO stages I to III & 70 & 70 & $\begin{array}{c}47.5 \\
(21-78)\end{array}$ & $\begin{array}{c}53.6 \\
(27-78)\end{array}$ & 4 \\
\hline $\begin{array}{l}\text { Morgan } \\
2007\end{array}$ & $R$ & Ireland & $\begin{array}{l}2000.09- \\
2005.06\end{array}$ & $\begin{array}{c}\text { FIGO stage } 1 \mathrm{a} \\
\text { to } 1 \mathrm{~b}\end{array}$ & 30 & 30 & $\begin{array}{c}38 \\
(20-63)\end{array}$ & $\begin{array}{c}35 \\
(25-54)\end{array}$ & 7 \\
\hline Naik 2010 & $\begin{array}{c}P \\
(R C T)\end{array}$ & UK & NA & FIGO stage IB1 & 7 & 6 & $\begin{array}{c}38.5 \\
(33.5-53.5)\end{array}$ & $\begin{array}{c}37 \\
(29.5-46)\end{array}$ & NA \\
\hline $\begin{array}{l}\text { Pahisa } \\
2010\end{array}$ & $P$ & Spain & $\begin{array}{c}1997.01- \\
2007.12\end{array}$ & FIGO stage IA-IIB & 67 & 23 & $\begin{array}{c}51 \\
(29-75)\end{array}$ & $\begin{array}{c}48 \\
(31-67)\end{array}$ & 7 \\
\hline $\begin{array}{l}\text { Papacha- } \\
\text { ralabous } \\
2009\end{array}$ & $R$ & UK & $\begin{array}{l}2003.01- \\
2006.06\end{array}$ & FIGO stage $1 \mathrm{~A} 2-1 \mathrm{~B}$ & 14 & 12 & $38.6 \pm 3.6$ & $43.5 \pm 12.9$ & 5 \\
\hline $\begin{array}{l}\text { Sharma } \\
2006\end{array}$ & $R$ & England & 1999-2005 & FIGO stage IA2-IIB & 27 & 28 & $\begin{array}{c}43.4 \\
(28-60)\end{array}$ & $\begin{array}{c}42.8 \\
(28-66)\end{array}$ & 6 \\
\hline Steed 2004 & $P$ & Canada & $\begin{array}{l}1996.11- \\
2003.12\end{array}$ & FIGO stage IA/IB & 71 & 205 & $\begin{array}{c}43 \\
(30-69)\end{array}$ & $\begin{array}{c}44 \\
(24-86)\end{array}$ & 6 \\
\hline Yu 2013 & $R$ & China & $\begin{array}{c}2003.12- \\
2008.12\end{array}$ & $\begin{array}{c}\text { FIGO stage IA } \\
\text { to IIA }\end{array}$ & 40 & 40 & $\begin{array}{c}44.9 \\
(30-61)\end{array}$ & $\begin{array}{c}39.1 \\
(28-57)\end{array}$ & 7 \\
\hline Zhang 2017 & $R$ & China & $\begin{array}{l}2008.03- \\
2012.07\end{array}$ & $\begin{array}{c}\text { FIGO stage IA2 } \\
\text { to IIB }\end{array}$ & 35 & 42 & $\begin{array}{c}45 \\
(29-64)\end{array}$ & $\begin{array}{c}46.6 \\
(27-75)\end{array}$ & 6 \\
\hline
\end{tabular}

$P$-prospective, $R$-retrospective, FIGO - International Federation of Gynaecology and Obstetrics, LARVH - laparoscopic-assisted radical vaginal hysterectomy, $A R H$ - abdominal radical hysterectomy, ${ }^{*}$ - mean (range), NOS - Newcastle-Ottawa Scale.

sensitivity analysis showed that the variation ranges of WMD $(95 \% \mathrm{Cl})$ for operation time (WMD [95\% Cl] $=47.64[35.45,59.84]$ to $55.45[44.67,66.23])$, blood loss (WMD [95\% Cl] $=-354.41[-458.60,-250.22]$ to $-270.72[-443.23,-98.21])$, and hospital stay (WMD $[95 \% \mathrm{Cl}]=-3.76[-5.15,-2.36]$ to $-2.81[-4.45$, $-1.16])$ were not significantly reversed, showing stable outcomes.

\section{Meta-analysis of complications}

Four articles reported the number of complications in both LARVH and ARH groups (Figure $4 \mathrm{~A}$ ). No significant heterogeneity was observed $\left(I^{2}=0.0 \%\right.$, $p=0.589$ ). Complications between LARVH and ARH were evaluated using the fixed effects model; no significant difference was found between the groups $(\mathrm{OR}=0.63,95 \% \mathrm{Cl}: 0.35,1.13, p=0.123)$. Further, subgroup analysis was performed for different types of research (prospective vs. retrospective), and the results were not reversed (Table II). Publication bias among the studies was not significant (Egger's test; $p=0.359$ ). Higher complication rates were observed in LARVH than in ARH, after removing a study [20], suggesting that the pooled result for overall complication rate analysis was unstable. 
Urinary tract infection was the most commonly reported complication in the included studies $(n=3)$. Pooled data showed that the potential risk for patients developing urinary tract infection was significantly lower in the LARVH group than in the ARH group $(\mathrm{OR}=0.34,95 \% \mathrm{Cl}: 0.13,0.89, p=0.028$; Figure $4 \mathrm{~B})$.

Subgroup analysis of research type showed no significant difference in the rate of urinary tract infection between ARH and LARVH in prospective $(\mathrm{OR}=0.07,95 \% \mathrm{Cl}: 0.00,1.67, p=0.100)$ and retrospective studies (OR $=0.42,95 \% \mathrm{Cl}: 0.15,1.18, p=$ 0.100 ; Table II). Sensitivity analysis showed that the outcome was unstable. When a study by Naik et al. [22] or Sharma et al. [25] was removed, no significant difference in the rate of urinary tract infections was found between the ARH and LARVH groups. In addition, Egger's test demonstrated that publication bias was not significant $(p=0.081)$.

\section{Meta-analysis of oncologic outcomes}

Seven studies reported cancer recurrence in patients after treatment. No significant heterogeneity was observed among them $\left(R^{2}=0.0 \%, p=0.859\right)$; the

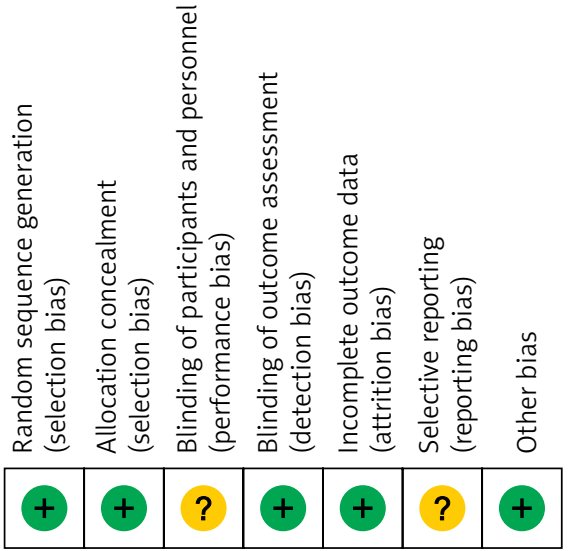

Figure 2. Risk-of-bias summary for the randomised control study included in this metaanalysis

"+" - low risk of bias; "?" - unclear risk of bias.

fixed effects model was used to pool data regarding recurrence in patients after treatment. Pooled results showed that patients who underwent LARVH had lower recurrence rates than patients who underwent ARH (OR $=0.549,95 \% \mathrm{Cl}: 0.302,0.998$, $p=0.049$; Figure $5 \mathrm{~A})$. However, subgroup analyses

A

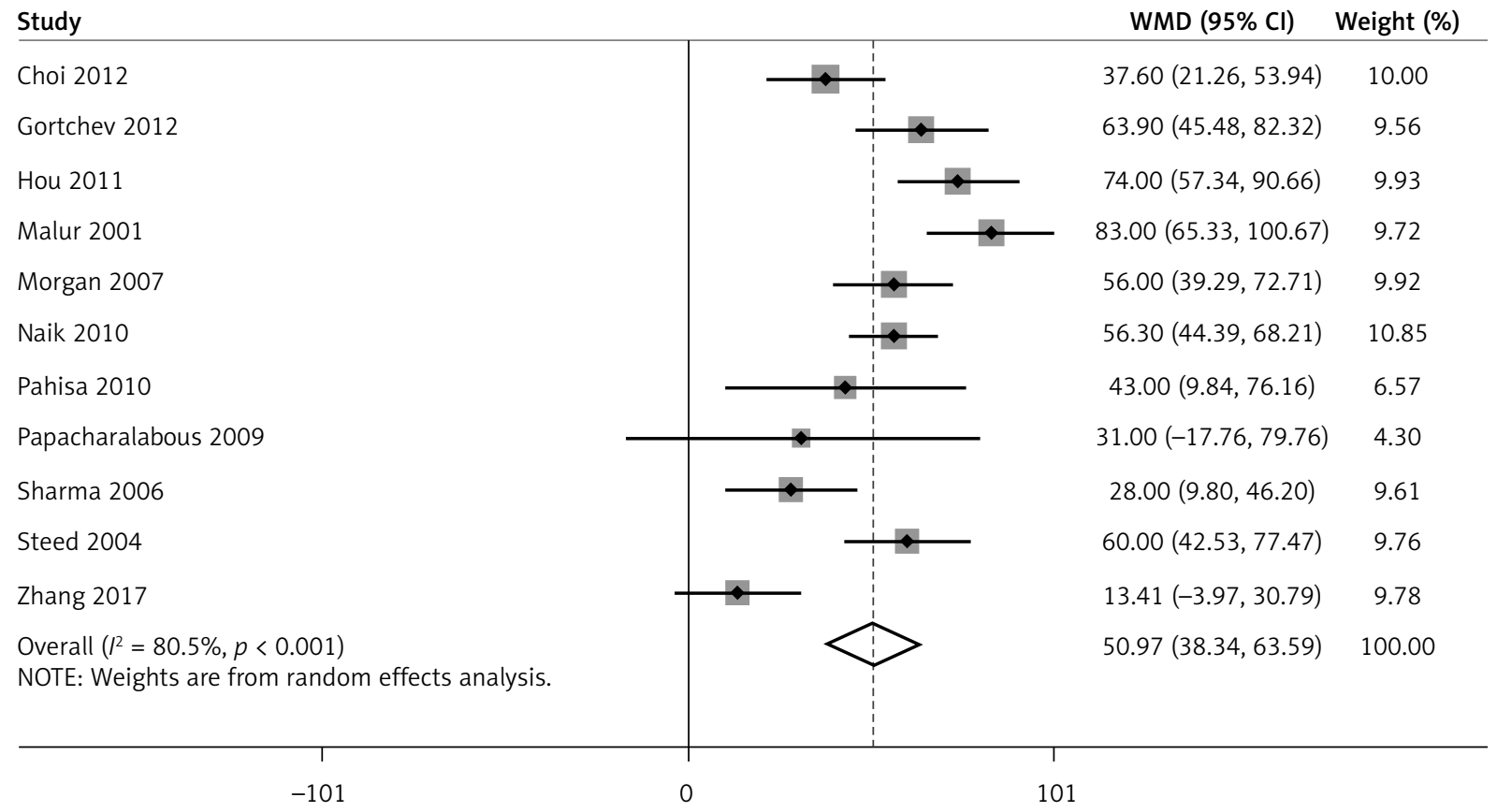

Figure 3. Pooled perioperative outcome comparisons between patients with cervical cancer who underwent laparoscopic-assisted radical vaginal hysterectomy and those who underwent abdominal radical hysterectomy. A - operation time 
B

Study

WMD $(95 \% \mathrm{Cl})$

Weight (\%)

Choi 2012

Hou 2011

Naik 2010

Papacharalabous 2009

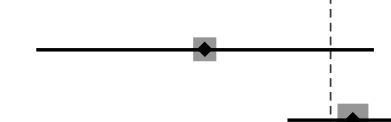

$-32.60(-104.23,39.03)$

18.28

$-555.35(-814.22,-296.48)$

13.21

$-384.70(-560.53,-208.87)$

15.76

Sharma 2006

$-713.00(-1256.42,-169.58)$

6.52

$-236.00(-447.43,-24.57)$

14.67

Steed 2004

Zhang 2017

Overall $\left(I^{2}=85.2 \%, p<0.001\right)$

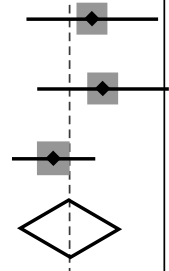

$-200.00(-415.06,15.06)$

14.56

$-359.05(-490.63,-227.47) \quad 17.00$

NOTE: Weights are from random effects analysis.

\begin{tabular}{ccc}
\hline 1256 & 0 & 1256
\end{tabular}

C

Study

WMD $(95 \% \mathrm{Cl})$

Weight (\%)

Choi 2012

Gortchev 2012

Hou 2011

Malur 2001

Morgan 2007

Naik 2010

Papacharalabous 2009

Steed 2004

Yu 2013

Overall $\left(I^{2}=96.3 \%, p<0.001\right)$

NOTE: Weights are from random effects analysis.

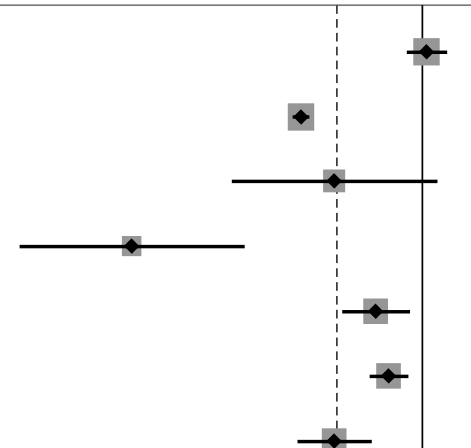

$0.10(-0.68,0.88) \quad 12.62$

$-4.80(-5.05,-4.55) \quad 12.95$

$-3.50(-7.50,0.50) \quad 7.25$

$-11.40(-15.81,-6.99) \quad 6.63$

$-1.90(-3.21,-0.59) \quad 11.97$

$-1.40(-2.11,-0.69) \quad 12.68$

$-3.50(-4.92,-2.08) \quad 11.81$

$-4.00(-4.99,-3.01) \quad 12.39$

$-3.80(-5.30,-2.30) \quad 11.70$

$-3.38(-5.00,-1.76) \quad 100.00$

$-15.8$

15.8

Figure 3. Cont. B - blood loss, C - hospital stay 
Table II. Outcomes of subgroup analyses

\begin{tabular}{|c|c|c|c|c|c|}
\hline \multirow[t]{2}{*}{ Outcomes } & \multirow{2}{*}{$\begin{array}{l}\text { No. of } \\
\text { studies }\end{array}$} & \multicolumn{2}{|c|}{ Heterogeneity test } & \multicolumn{2}{|l|}{ Effect value } \\
\hline & & $I^{2}$ & $P$-value & WMD/OR $(95 \% \mathrm{Cl})$ & $P$-value \\
\hline Operation time (overall): & 11 & $80.5 \%$ & $<0.001$ & $50.97(38.34,63.59)$ & $<0.001$ \\
\hline \multicolumn{6}{|l|}{ Study type: } \\
\hline Prospective & 5 & $60.6 \%$ & 0.038 & $55.46(43.04,67.88)$ & $<0.001$ \\
\hline Retrospective & 6 & $87.2 \%$ & $<0.001$ & $46.99(24.30,69.88)$ & $<0.001$ \\
\hline \multicolumn{6}{|l|}{ Area: } \\
\hline Asia & 3 & $91.9 \%$ & $<0.001$ & $41.73(7.57,75.90)$ & 0.017 \\
\hline Western & 8 & $65.9 \%$ & 0.005 & $55.49(43.72,67.25)$ & $<0.001$ \\
\hline Blood loss (overall): & 7 & $85.2 \%$ & $<0.001$ & $-311.21(-482.77,-139.64)$ & $<0.001$ \\
\hline \multicolumn{6}{|l|}{ Study type: } \\
\hline Prospective & 4 & $88.4 \%$ & $<0.001$ & $-277.39(-520.66,-34.11)$ & 0.025 \\
\hline Retrospective & 3 & $29.1 \%$ & 0.244 & $-342.29(-482.77,-139.64)$ & $<0.001$ \\
\hline \multicolumn{6}{|l|}{ Area: } \\
\hline Asia & 3 & $93.1 \%$ & $<0.001$ & $-297.45(-596.71,1.82)$ & 0.051 \\
\hline Western & 4 & $29.9 \%$ & 0.233 & $-309.51(-450.25,-168.76)$ & $<0.001$ \\
\hline Hospital stay (overall): & 9 & $96.3 \%$ & $<0.001$ & $-3.38(-5.00,-1.76)$ & $<0.001$ \\
\hline \multicolumn{6}{|l|}{ Study type: } \\
\hline Prospective & 4 & $92.8 \%$ & $<0.001$ & $-1.98(-3.93,-0.02)$ & 0.047 \\
\hline Retrospective & 5 & $87.1 \%$ & $<0.001$ & $-4.22(-5.77,-2.66)$ & $<0.001$ \\
\hline \multicolumn{6}{|l|}{ Area: } \\
\hline Asia & 3 & $91.1 \%$ & $<0.001$ & $-2.21(-5.38,0.97)$ & 0.173 \\
\hline Western & 6 & $95.2 \%$ & $<0.001$ & $-3.82(-5.47,-2.16)$ & $<0.001$ \\
\hline Perioperative complications: & 4 & $0.0 \%$ & 0.589 & $0.63(0.35,1.13)$ & 0.123 \\
\hline \multicolumn{6}{|l|}{ Study type: } \\
\hline Prospective & 1 & - & - & $0.40(0.13,1.22)$ & 0.108 \\
\hline Retrospective & 3 & $0.0 \%$ & 0.591 & $0.73(0.37,1.46)$ & 0.376 \\
\hline Urinary tract infection: & 3 & $0.0 \%$ & 0.479 & $0.34(0.13,0.89)$ & 0.028 \\
\hline \multicolumn{6}{|l|}{ Study type: } \\
\hline Prospective & 1 & - & - & $0.07(0.00,1.67)$ & 0.100 \\
\hline Retrospective & 2 & $0.0 \%$ & 0.582 & $0.42(0.15,1.18)$ & 0.100 \\
\hline Recurrence (Overall): & 7 & $0.0 \%$ & 0.859 & $0.549(0.302,0.998)$ & 0.049 \\
\hline \multicolumn{6}{|l|}{ Study type: } \\
\hline Prospective & 1 & - & - & $0.42(0.09,2.05)$ & 0.085 \\
\hline Retrospective & 6 & $0.0 \%$ & 0.785 & $0.57(0.30,1.08)$ & 0.286 \\
\hline \multicolumn{6}{|l|}{ Area: } \\
\hline Asia & 2 & $0.0 \%$ & 0.859 & $0.48(0.17,1.33)$ & 0.156 \\
\hline Western & 5 & $0.0 \%$ & 0.864 & $0.59(0.28,1.23)$ & 0.160 \\
\hline Death (Overall): & 4 & $0.0 \%$ & 0.593 & $0.52(0.22,1.20)$ & 0.124 \\
\hline \multicolumn{6}{|l|}{ Study type: } \\
\hline Prospective & 1 & - & - & $0.32(0.04,2.44)$ & 0.273 \\
\hline Retrospective & 3 & $0.0 \%$ & 0.444 & $0.56(0.22,1.39)$ & 0.211 \\
\hline \multicolumn{6}{|l|}{ Area: } \\
\hline Asia & 1 & - & - & $0.58(0.10,3.35)$ & 0.539 \\
\hline Western & 3 & $0.0 \%$ & 0.388 & $0.50(0.19,1.31)$ & 0.158 \\
\hline
\end{tabular}




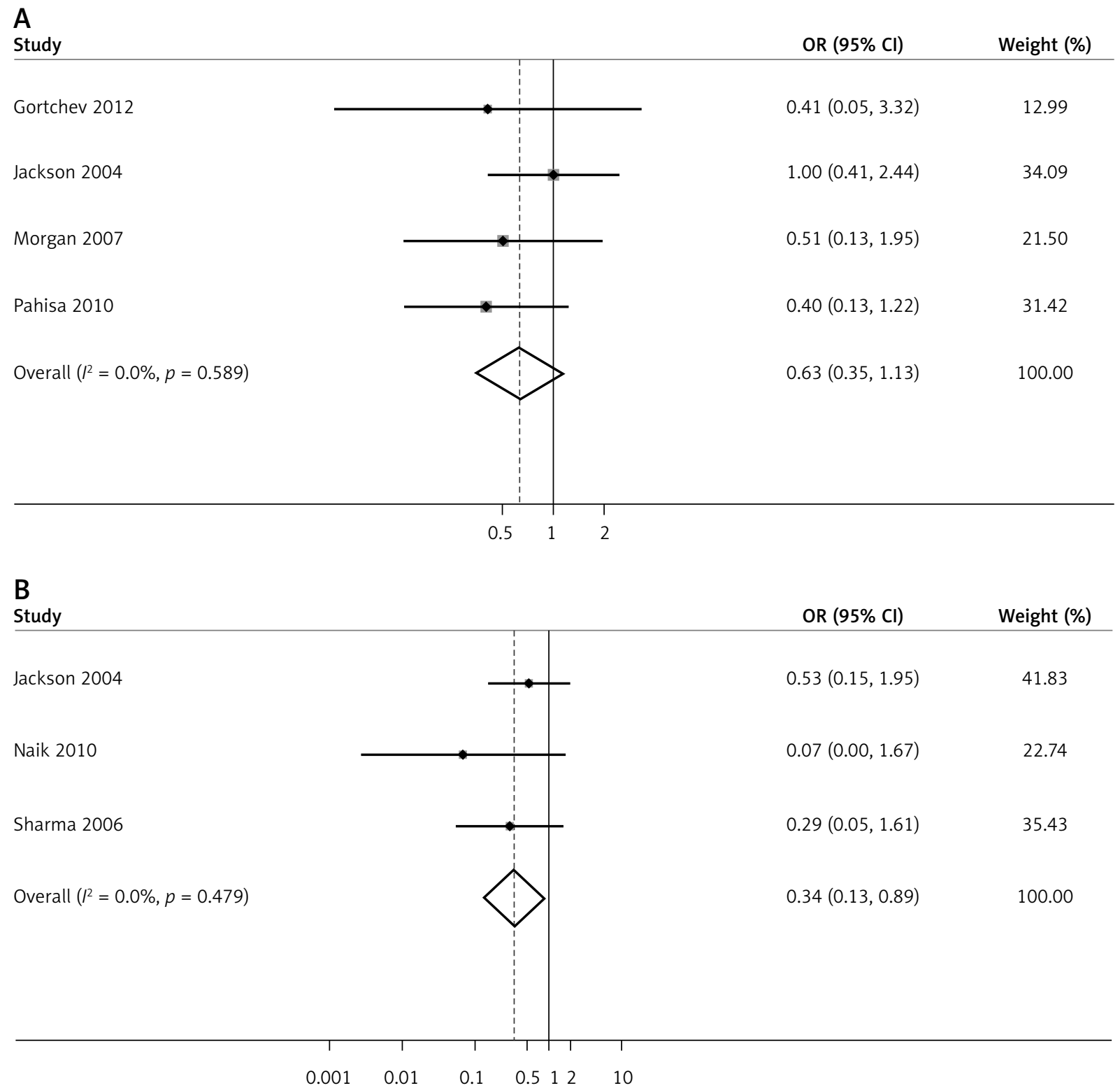

Figure 4. Estimated complications between laparoscopic-assisted radical vaginal hysterectomy and abdominal radical hysterectomy in treating cervical cancer. A - perioperative complications, $\mathbf{B}$ - urinary tract infection

of research type and region identified no significant differences between these groups, which conflicted with the pooled results and indicated an unstable outcome. Similarly, sensitivity analysis showed that the outcome was unstable. After removing the study by Gortchev et al. [18], Pahisa et al. [23], or Zhang et al. [14], cancer recurrence rates no longer significantly differed between patients who underwent LARVH and ARH. Meanwhile, publication bias among these studies was significant $(p=0.017)$.
Four studies reported patient deaths after treatment. No significant heterogeneity was observed $\left(I^{2}=0.0 \%, p=0.593\right)$; thus a fixed effects model was used to pool data on mortality rate. The results indicated no significant difference in postoperative mortality between the groups ( $\mathrm{OR}=0.52,95 \% \mathrm{Cl}$ : $0.22,1.20, p=0.124$, Figure $5 \mathrm{~B}$ ). Subgroup analyses of research types revealed no significant differences between LARVH and ARH with regard to mortality in prospective $(\mathrm{OR}=0.32,95 \% \mathrm{Cl}: 0.04,2.44, p=0.273)$ 


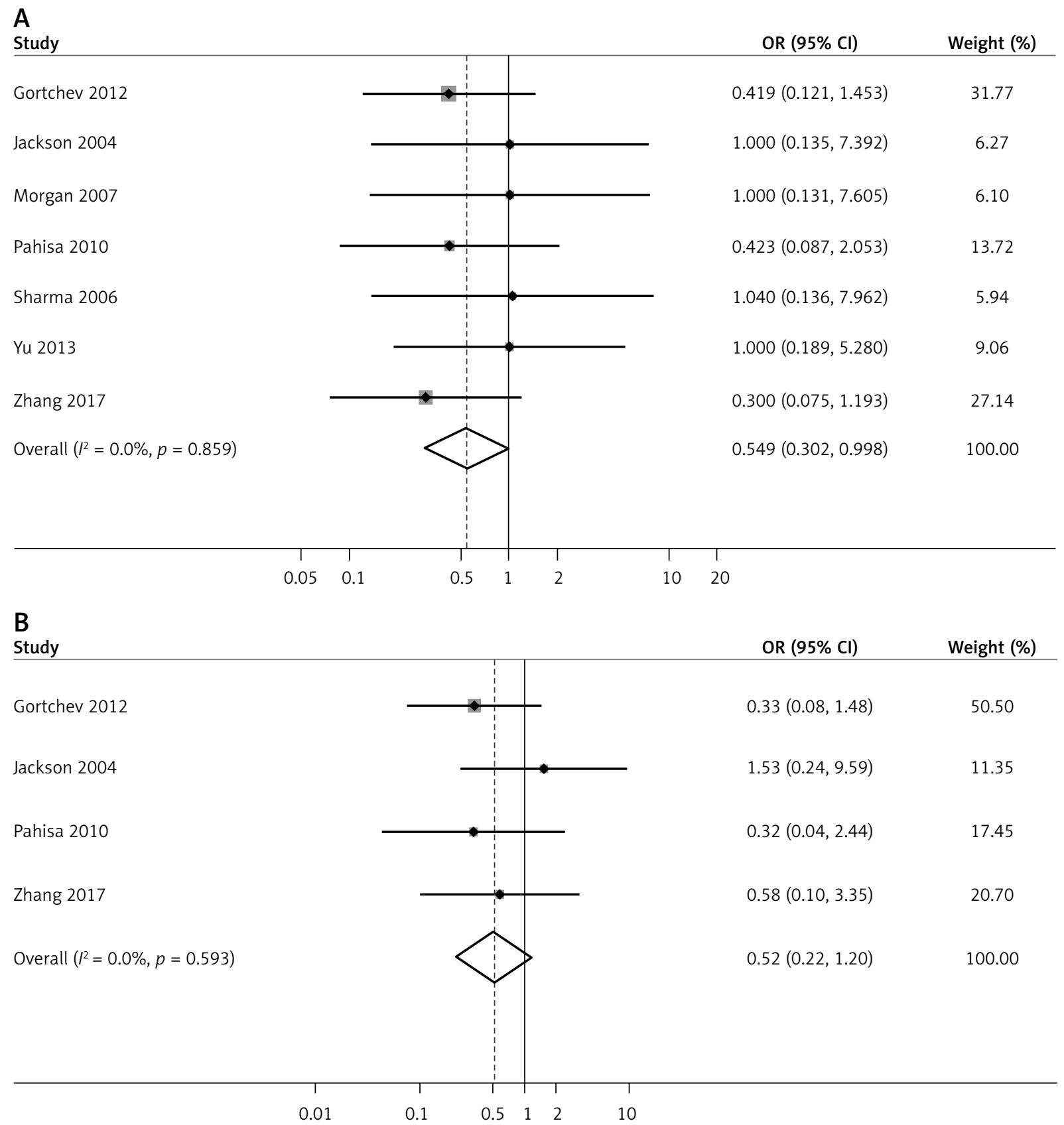

Figure 5. Forest plots of comparison of postoperative outcomes between laparoscopic-assisted radical vaginal hysterectomy and abdominal radical hysterectomy in treating cervical cancer: $\mathbf{A}$ - recurrence, $\mathbf{B}$ - mortality rate

and retrospective studies $(\mathrm{OR}=0.56,95 \% \mathrm{Cl}: 0.22$, 1.39, $p=0.211$ ). Moreover, no significant differences were identified with regard to the mortality of patients undergoing LARVH and ARH in Asian $(\mathrm{OR}=0.58,95 \% \mathrm{Cl}: 0.10,3.35, p=0.539)$ and Western countries $(\mathrm{OR}=0.50,95 \% \mathrm{Cl}: 0.19,1.31, p=$ 0.158 , Table II). No significant publication bias was observed ( $p=0.691)$. Sensitivity analysis showed that the pooled results of postoperative mortality assessment were stable $(\mathrm{OR}[95 \% \mathrm{Cl}]=0.39[0.14$, $1.05]$ to 0.71 [0.24, 2.03]) after removing each study.

We further compared the number of patients with lymph node metastasis using a fixed effects model $\left(I^{2}=0.0 \%, p=0.739\right)$, and the results revealed 
A

Study

OR $(95 \% \mathrm{Cl})$

Weight (\%)

Choi 2012

Gortchev 2012

Jackson 2004

Morgan 2007

Naik 2010

Pahisa 2010

Papacharalabous 2009

Sharma 2006

Steed 2004

Yu 2013

Overall $\left(I^{2}=0.0 \%, p=0.739\right)$

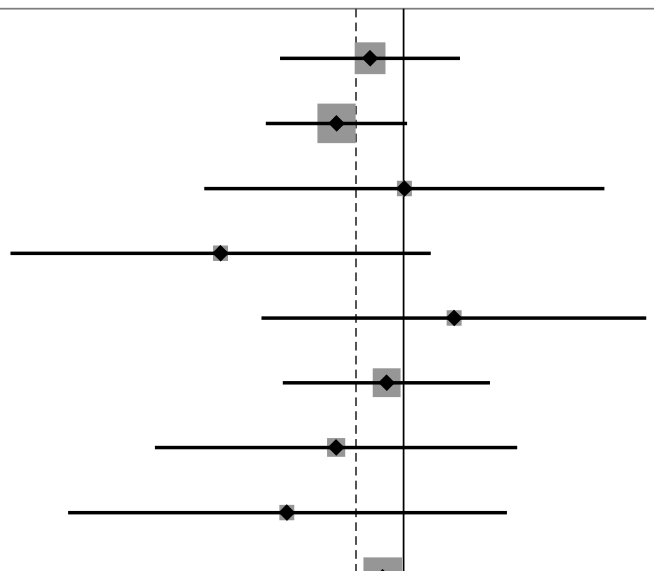

$0.62(0.17,2.19) \quad 16.34$

$0.39(0.14,1.04) \quad 26.55$

$1.00(0.06,16.44) \quad 3.33$

$0.08(0.00,1.44) \quad 3.01$

$2.00(0.13,29.81) \quad 3.57$

$0.78(0.18,3.30) \quad 12.51$

$0.38(0.03,4.87) \quad 4.05$

$0.19(0.01,4.21) \quad 2.74$

$0.74(0.27,2.07) \quad 24.86$

$0.08(0.00,1.49) \quad 3.04$

$0.52(0.31,0.87) \quad 100.00$

\begin{tabular}{c|c|c}
\hline & & \\
\hline 0.00401 & 1 & 249
\end{tabular}

\section{B}

Study

WMD $(95 \% \mathrm{Cl}) \quad$ Weight (\%)

Choi 2012

Gortchev 2012

Hou 2011

Malur 2001

Morgan 2007

Naik 2010

Pahisa 2010

Papacharalabous 2009

Sharma 2006

Yu 2013

Zhang 2017

Overall $\left(I^{2}=97.4 \%, p<0.001\right)$

NOTE: Weights are from random effects analysis.

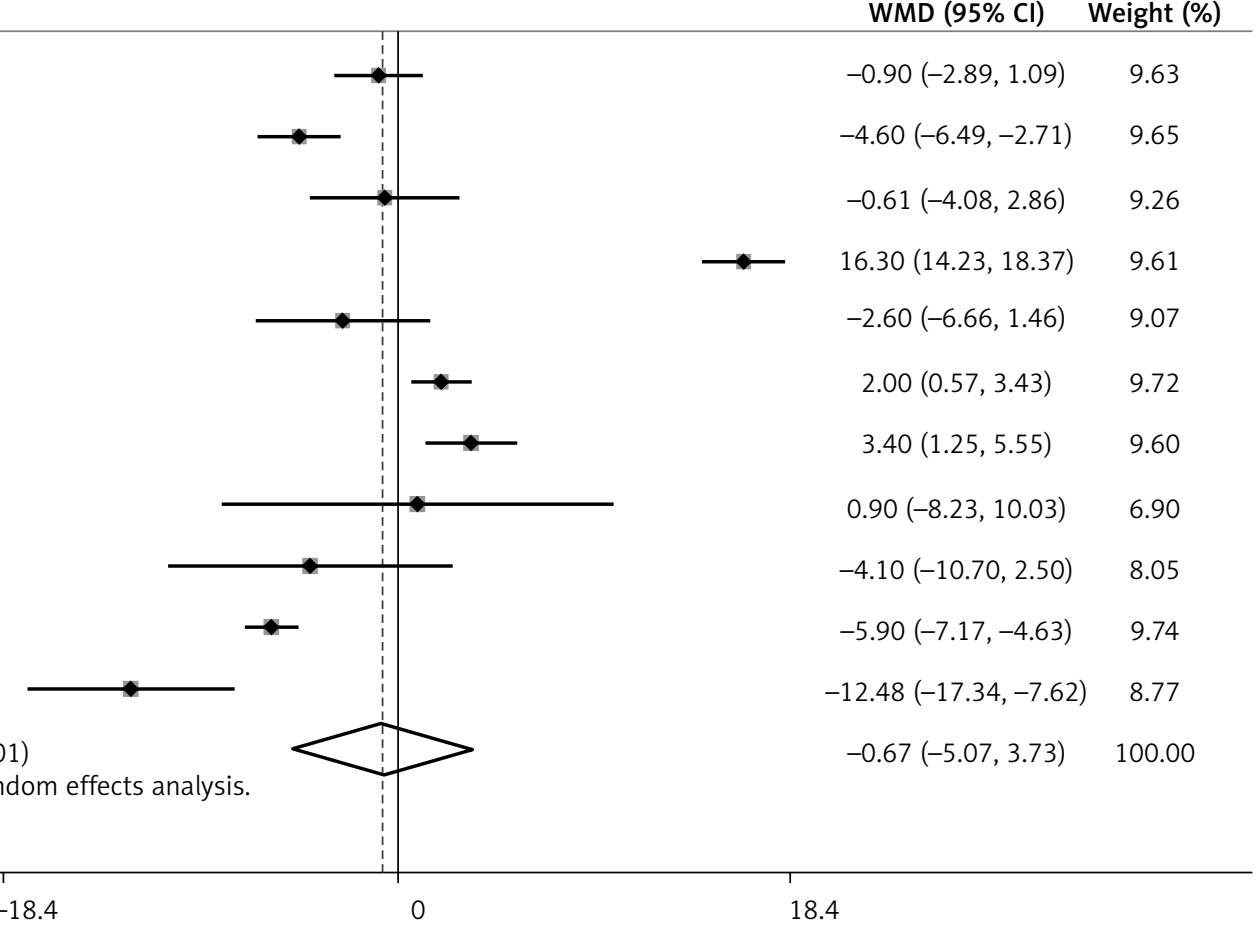

Figure 6. Forest plots of comparison of lymph nodes and patients requiring adjuvant radiotherapy between laparoscopic-assisted radical vaginal hysterectomy and abdominal radical hysterectomy in treating cervical cancer. $\mathbf{A}$ - number of patients with lymph node metastasis, $\mathbf{B}$ - number of removed lymph nodes 


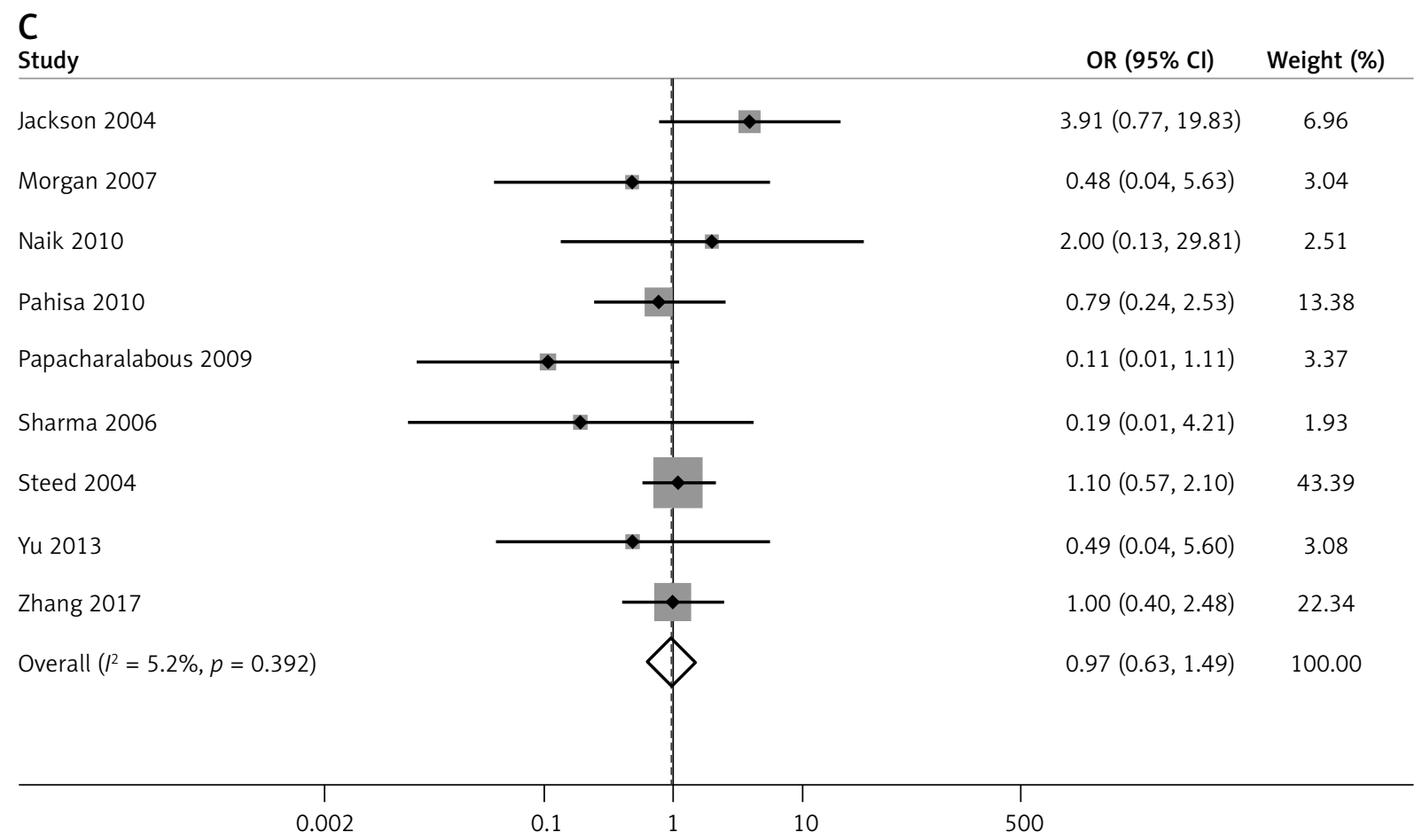

Figure 6. Cont. C - patients requiring adjuvant radiotherapy

a significant difference between the two groups (OR $=0.52,95 \% \mathrm{Cl}: 0.31,0.87, p=0.013$, Figure $6 \mathrm{~A})$. As for the number of removed lymph nodes, significant heterogeneity was observed among studies $\left(R^{2}=\right.$ $97.4 \%, p<0.001)$; therefore a random effects model was used to pool the results. The results indicated no significant difference in the number removed lymph nodes between the groups $(O R=-0.67$, $95 \% \mathrm{Cl}:-5.07,3.73, p=0.765$, Figure $6 \mathrm{~B})$. Additionally, the percentage of patients requiring adjuvant radiotherapy was analysed based on a fixed effects model $\left(I^{2}=5.2 \%, p=0.392\right)$, and no significant difference was found between the two groups (OR = $0.97,95 \% \mathrm{Cl}: 0.63,1.49, p=0.886$, Figure $6 \mathrm{C})$.

\section{Discussion}

This study aimed to compare LARVH with ARH for patients with cervical cancer and to systematically analyse data in published studies. In total, 579 patients who underwent LARVH and 810 who underwent $A R H$ were included. Pooled data showed that although $L A R V H$ required a longer operation time than $\mathrm{ARH}$, it was superior to $\mathrm{ARH}$, with lower bleeding volume and shorter hospital stay. No significant difference in total complications was found.
The risk of urinary tract infections was significantly lower in patients who underwent LARVH. This study suggested that LARVH might be superior to ARH in treating cervical cancer, with lower bleeding volume, shorter hospital stay, and lower risk of urinary tract infection.

Most published individual studies have supported the longer operating time of LARVH than that of $\mathrm{ARH}[28,29]$. Although our results were consistent with those of previous studies in terms of the longer operation time of LARVH than of ARH, significant heterogeneity was observed among the included studies that focused on operation time, surgical bleeding, and length of hospital stay. Subsequently, subgroup analysis stratified by study type and region was performed; heterogeneity continued to exist. LARVH requires more skill and experience than $\mathrm{ARH}[30,31]$. Moreover, vaginal cuff identification requires superior tactile and purely intuitive skills. Thus, with advances in laparoscopy and increasing experience of surgeons, operating time may no longer be a limitation of LARVH. We speculated that heterogeneity is introduced by differences in the degree of skill of surgeons and patient background. Prospective research adjusting patient background and surgeon experience is required to confirm the 
Table III. Surgical related information

\begin{tabular}{|c|c|c|c|c|c|c|c|c|c|c|}
\hline Study & $\begin{array}{l}\text { The } \\
\text { Same } \\
\text { time } \\
\text { period }\end{array}$ & Group & $N$ & FIGO stage & $\begin{array}{l}\text { Lymph } \\
\text { node me- } \\
\text { tastasis }\end{array}$ & $\begin{array}{c}\text { Patients } \\
\text { treated } \\
\text { with ad- } \\
\text { juvant } \\
\text { radio- } \\
\text { therapy }\end{array}$ & $\begin{array}{l}\text { Rate of } \\
\text { positive } \\
\text { margins }\end{array}$ & $\begin{array}{l}\text { Removed } \\
\text { lymph } \\
\text { nodes }\end{array}$ & $\begin{array}{l}\text { Team of } \\
\text { operations }\end{array}$ & $\begin{array}{l}\text { Type of } \\
\text { surgery }\end{array}$ \\
\hline \multirow[t]{2}{*}{$\begin{array}{l}\text { Choi } \\
2012\end{array}$} & \multirow[t]{2}{*}{ Yes } & LARVH & 89 & $\begin{array}{c}3 / 11 / 73 / 2(\mid \mathrm{A} 1 / \\
\mid \mathrm{A} 2 / \mathrm{B} 1 / \mathrm{IA})\end{array}$ & $4(4.5 \%)$ & 0 & 0 & $19.5 \pm 6.2$ & $\begin{array}{l}1 \text { surgeon } \\
\text { (DSB) }\end{array}$ & $3 \mathrm{II}, 86 \mathrm{III}$ \\
\hline & & $\mathrm{ARH}$ & 99 & $\begin{array}{c}\text { 1/8/83/7 (IA1/ } \\
\text { |A2/IB1/IIA) }\end{array}$ & 7 (7.1\%) & 0 & 0 & $20.4 \pm 7.7$ & $\begin{array}{c}1 \text { surgeon } \\
(\mathrm{JHL})\end{array}$ & 9 II, 90 III \\
\hline \multirow{2}{*}{$\begin{array}{l}\text { Gortch- } \\
\text { ev } 2012\end{array}$} & \multirow[t]{2}{*}{ Yes } & LARVH & 46 & 46 IB1 & $5(10.9 \%) /$ & $N R$ & NR & $11.3 \pm 5.2$ & 1 surgeon & \multirow[t]{2}{*}{ Type III } \\
\hline & & ARH & 175 & 175 IB1 & $42(24.0 \%)$ & NR & $N R$ & $15.9 \pm 7.7$ & 2 surgeon & \\
\hline \multirow[t]{2}{*}{$\begin{array}{l}\text { Hou } \\
2011\end{array}$} & \multirow[t]{2}{*}{ Yes } & LARVH & 33 & $\begin{array}{c}\text { 4/10/15/4 (IA/ } \\
\text { IB/IIA/IIB) }\end{array}$ & 0 & NR & NR & $19.74 \pm 7.43$ & \multirow{2}{*}{$\begin{array}{l}\text { The same } \\
\text { group of } \\
\text { surgeons }\end{array}$} & \multirow[t]{2}{*}{ NR } \\
\hline & & $\mathrm{ARH}$ & 30 & $\begin{array}{c}2 / 10 / 14 / 4(\mid \mathrm{A} / \\
\mathrm{IB} / \mathrm{IIA} / \mathrm{IIB})\end{array}$ & 0 & NR & NR & $20.35 \pm 6.62$ & & \\
\hline \multirow{2}{*}{$\begin{array}{l}\text { Jack- } \\
\text { son } \\
2004\end{array}$} & \multirow[t]{2}{*}{ Yes } & LARVH & 50 & $\begin{array}{c}\text { 2/47/1 (IA2/IB1/ } \\
\text { IB2) }\end{array}$ & $1(2 \%)$ & $7(14 \%)$ & $5(10 \%)$ & Median 15 & \multirow{2}{*}{$\begin{array}{l}\text { The same } \\
\text { group of } \\
\text { surgeons }\end{array}$} & \multirow[t]{2}{*}{ NR } \\
\hline & & $\mathrm{ARH}$ & 50 & $\begin{array}{c}2 / 47 / 1(|A 2 /| B 1 / \\
\text { IB2) }\end{array}$ & $1(2 \%)$ & $2(4 \%)$ & $1(2 \%)$ & Median 16 & & \\
\hline \multirow[t]{2}{*}{$\begin{array}{l}\text { Malur } \\
2001\end{array}$} & \multirow[t]{2}{*}{ No } & LARVH & 70 & $\begin{array}{c}3 / 13 / 41 / 3 / 9 / 1 / 0 \\
(|\mathrm{~A} 1 / \mathrm{IA} 2 / \mathrm{IB} /| \mathrm{II} / \\
\text { IIB/IIIA/IIIB) }\end{array}$ & NR & NR & NR & $27(10-56)^{\#}$ & \multirow[t]{2}{*}{$\begin{array}{l}\text { Senior } \\
\text { registrars }\end{array}$} & \multirow[t]{2}{*}{ Type II } \\
\hline & & $\mathrm{ARH}$ & 70 & $\begin{array}{c}1 / 1 / 51 / 5 / 11 / 0 / 1 \\
(|\mathrm{~A} 1 /| \mathrm{A} 2 / \mathrm{IB} / \| \mathrm{A} / \\
\text { IIB/IIIA/IIIB) }\end{array}$ & NR & $100 \%$ & NR & $10.7(0-26)$ & & \\
\hline \multirow{2}{*}{$\begin{array}{l}\text { Mor- } \\
\text { gan } \\
2007\end{array}$} & \multirow[t]{2}{*}{ Yes } & LARVH & 30 & $\begin{array}{c}\text { 9/21/0/0 (IA/IB/ } \\
\text { IIA/IIB) }\end{array}$ & 0 & 1 (3.3\%) & NR & $14.8(3-37)^{\#}$ & \multirow{2}{*}{$\begin{array}{l}\text { The same } \\
\text { group of } \\
\text { surgeons }\end{array}$} & \multirow[t]{2}{*}{ Type III } \\
\hline & & $\mathrm{ARH}$ & 30 & $\begin{array}{c}2 / 24 / 2 / 2(\mathrm{IA} / \mathrm{IB} / \\
\| \mathrm{I} / \mathrm{IIB})\end{array}$ & $5(16.7 \%)$ & $2(6.7 \%)$ & NR & $17.4(6-36)$ & & \\
\hline \multirow[t]{2}{*}{$\begin{array}{l}\text { Naik } \\
2010\end{array}$} & \multirow[t]{2}{*}{ Yes } & LARVH & 7 & $7 \mid \mathrm{B} 1$ & $2(28.57 \%)$ & $\begin{array}{c}2 \\
(28.57 \%)\end{array}$ & $\begin{array}{c}1 \\
(14.29 \%)\end{array}$ & $14(12-19)^{\#}$ & \multirow{2}{*}{$\begin{array}{l}\text { The same } \\
\text { group of } \\
\text { surgeons }\end{array}$} & \multirow[t]{2}{*}{ NR } \\
\hline & & $\mathrm{ARH}$ & 6 & 6 IB1 & $1(16.7 \%)$ & $1(16.7 \%)$ & 0 (0.0\%) & $12(11-14)$ & & \\
\hline \multirow[t]{2}{*}{$\begin{array}{l}\text { Pahisa } \\
2010\end{array}$} & \multirow[t]{2}{*}{ No } & LARVH & 67 & $\begin{array}{c}\text { 3/61/3 (IA2/IB1/ } \\
\text { IIA) }\end{array}$ & 7 (10.4\%) & $\begin{array}{c}12 \\
(17.9 \%)\end{array}$ & 0 & $\begin{array}{c}15.8 \\
(10-33)^{\#}\end{array}$ & \multirow[t]{2}{*}{$\begin{array}{l}\text { J.P, S.M-R, } \\
\text { or A.T. }\end{array}$} & \multirow[t]{2}{*}{ NR } \\
\hline & & ARH & 23 & $\begin{array}{c}2 / 21 / 0(\mid \mathrm{A} 2 / \mathrm{IB} 1 / \\
\text { IIA) }\end{array}$ & 3 (13.0\%) & $\begin{array}{c}5 \\
(21.7 \%) \\
\end{array}$ & 0 & $12.4(8-27)$ & & \\
\hline \multirow{2}{*}{$\begin{array}{l}\text { Pa- } \\
\text { pacha- } \\
\text { ral- } \\
\text { abous } \\
2009 \\
\end{array}$} & \multirow[t]{2}{*}{ Yes } & LARVH & 14 & $14(\mathrm{IA} 2-\mathrm{IB})$ & $1(7.7 \%)$ & $1(7.7 \%)$ & 0 & $22.4 \pm 10.6$ & S.BM & \multirow[t]{2}{*}{ NR } \\
\hline & & ARH & 12 & $12(\mathrm{IA} 2-\mid \mathrm{B})$ & 2 (16.7\%) & $\begin{array}{c}5 \\
(41.67 \%)\end{array}$ & 0 & $21.5 \pm 12.8$ & $\begin{array}{l}\text { A.T or } \\
\text { S.BM }\end{array}$ & \\
\hline Shar- & Yes & LARVH & 27 & 27/0 (IB1/IB2) & $0(0.0 \%)$ & $0(0.0 \%)$ & 0 & $23.5(7-62)^{*}$ & J.BM & NR \\
\hline $\begin{array}{l}\text { ma } \\
2006\end{array}$ & & $\mathrm{ARH}$ & 28 & 23/5 (IB1/IB2) & $2(7.1 \%)$ & $2(7.1 \%)$ & 0 & $27.6(13-57)$ & $\begin{array}{l}\text { J.BM and } \\
\text { R.SA }\end{array}$ & \\
\hline $\begin{array}{l}\text { Steed } \\
2004\end{array}$ & Yes & LARVH & 71 & $\begin{array}{c}\text { 14/10/46/1 (IA1/ } \\
\text { IA2/IB1/IB2) }\end{array}$ & $5(7.0 \%)$ & $\begin{array}{c}16 \\
(22.5 \%)\end{array}$ & $1(1.4 \%)$ & NR & $\begin{array}{l}\text { One sur- } \\
\text { geon }\end{array}$ & NR \\
\hline & & $\mathrm{ARH}$ & 205 & $\begin{array}{c}\text { 29/11/148/17 } \\
(\text { IA1/IA2/IB1/ } \\
\text { IB2) }\end{array}$ & 19 (9.3\%) & $\begin{array}{c}43 \\
(21.0 \%)\end{array}$ & $6(2.9 \%)$ & NR & $\begin{array}{l}\text { Six sur- } \\
\text { geons }\end{array}$ & \\
\hline
\end{tabular}


Table III. Cont.

\begin{tabular}{|c|c|c|c|c|c|c|c|c|c|c|}
\hline Study & $\begin{array}{l}\text { The } \\
\text { Same } \\
\text { time } \\
\text { period }\end{array}$ & Group & $N$ & FIGO stage & $\begin{array}{l}\text { Lymph } \\
\text { node me- } \\
\text { tastasis }\end{array}$ & $\begin{array}{c}\text { Patients } \\
\text { treated } \\
\text { with ad- } \\
\text { juvant } \\
\text { radio- } \\
\text { therapy }\end{array}$ & $\begin{array}{l}\text { Rate of } \\
\text { positive } \\
\text { margins }\end{array}$ & $\begin{array}{l}\text { Removed } \\
\text { lymph } \\
\text { nodes }\end{array}$ & $\begin{array}{c}\text { Team of } \\
\text { operations }\end{array}$ & $\begin{array}{l}\text { Type of } \\
\text { surgery }\end{array}$ \\
\hline \multirow[t]{2}{*}{$\begin{array}{l}\text { Yu } \\
2013\end{array}$} & Yes & LARVH & 40 & $\begin{array}{c}12 / 17 / 9 / 2(\mathrm{IA} 2 / \\
\text { IB1/IB2/IIA) }\end{array}$ & $0(0.0 \%)$ & $1(2.5 \%)$ & 0 & $\begin{array}{c}21.4 \\
(18-28)^{\#}\end{array}$ & \multirow{2}{*}{$\begin{array}{l}\text { The same } \\
\text { group of } \\
\text { surgeons }\end{array}$} & \multirow[t]{2}{*}{$N R$} \\
\hline & & $\mathrm{ARH}$ & 40 & $\begin{array}{c}\text { 6/15/13/6 (IA2/ } \\
\text { IB1/IB2/IIA) }\end{array}$ & $5(12.5 \%)$ & $2(5.0 \%)$ & 0 & $27.3(19-32)$ & & \\
\hline \multirow[t]{2}{*}{$\begin{array}{l}\text { Zhang } \\
2017\end{array}$} & Yes & LARVH & 35 & $\begin{array}{c}4 / 13 / 10 / 6 / 2 \\
(\mid \mathrm{A} 2 / \mathrm{IB} 1 / \mathrm{IB} 2 / \\
\text { IIA/IIB) }\end{array}$ & NR & $\begin{array}{c}20 \\
(57.14 \%)\end{array}$ & $N R$ & $23.71 \pm 9.45$ & \multirow{2}{*}{$\begin{array}{l}\text { The same } \\
2 \text { gynae- } \\
\text { cological } \\
\text { oncology } \\
\text { surgeons }\end{array}$} & \multirow[t]{2}{*}{ Type III } \\
\hline & & $\mathrm{ARH}$ & 42 & $\begin{array}{c}2 / 20 / 8 / 8 / 4 \\
(\mathrm{IA} 2 / \mathrm{IB} 1 / \mathrm{IB} 2 / \\
\text { IIA/IIB) }\end{array}$ & NR & $\begin{array}{c}24 \\
(57.14 \%)\end{array}$ & NR & $\begin{array}{c}36.19 \\
\pm 12.28\end{array}$ & & \\
\hline
\end{tabular}

conclusion of the comparison in operation time, surgical bleeding, and length of hospital stay between LARVH and ARH.

All publications assessing complications between open and laparoscopic surgery supported similar frequency of complications [18, 23]. However, some researchers questioned the clinical efficacy of LARVH compared to that of ARH [32]. LARVH was more suitable in small tumours, as they required less radical resection and owing to the complication that might occur by parametrial removal [22]. The complication rate ranged from $7.8 \%$ to $59 \%$, which might be attributed to the different criteria used for defining the complications and the different follow-up periods [33]. Therefore, the extent of parametrial removal and complications should be verified by further studies.

The aim of all treatment strategies is tumour removal with sufficient tumour-free margins, and local control is affected by radical surgery quality. Thus, early surgical treatment and preoperative care are important for patients with cervical cancer $[6,21]$. It is important to determine the most successful surgical treatment along with the associated recurrence rate and assessment of postoperative mortality. Our results showed that compared to patients who underwent $\mathrm{ARH}$, those who underwent LARVH had a lower cancer recurrence rate, and no significant difference was found in postoperative mortality. Thus, LARVH had a clinical efficacy equivalent to that of $\mathrm{ARH}$.

This study has some limitations. Firstly, articles published in English and limited electronic databases were searched as the data source; thus, some useful information might have been missed. Secondly, only one study was designed as a randomised control study. Among included studies, five were prospective and eight were retrospective. Retrospective studies might limit the strength of the conclusion. Thirdly, most of the patients in the included studies had cervical cancer at FIGO IA-IB1, but there were also fewer patients with disease at IB2, IIA, IIB, IIIA and IIIB stages (Table III), which might contribute to the heterogeneity in this meta-analysis.

\section{Conclusions}

Patients with cervical cancer who undergo LARVH would benefit from lower blood loss and shorter hospital stay. However, owing to the limited sample size and evaluation indicators, the better treatment option between LARVH and ARH should be determined by further clinical evidence.

\section{Conflict of interest}

The authors declare no conflict of interest.

\section{References}

1. Olorunfemi G, Ndlovu N, Masukume G, et al. Temporal trends in the epidemiology of cervical cancer in South Africa (1994-2012). Int J Cancer 2018; 143: 2238-49.

2. Pedersen K, Fogelberg S, Thamsborg LH, et al. An overview of cervical cancer epidemiology and prevention in Scandinavia. Acta Obstet Gynecol Scand 2018; 97: 795-807.

3. Daniyal M, Akhtar N, Ahmad S, et al. Update knowledge on cervical cancer incidence and prevalence in Asia. Asian Pac J Cancer Prev 2015; 16: 3617-20. 
4. Calandra D, Curia JD, Skalej E. Wertheim-Schauta operation in the surgical treatment of carcinoma of the cervix uteri. Sem Med 1963; 123: 373-4.

5. Li X, Li J, Wen H, et al. The survival rate and surgical morbidity of abdominal radical trachelectomy versus abdominal radical hysterectomy for stage IB1 cervical cancer. Ann Surg Oncol 2016; 23: 2953-8.

6. Bojahr B, Lober R, Straube W, et al. Gasless laparoscopic-assisted radical vaginal hysterectomy with lymphadenectomy for cervical carcinoma. J Am Assoc Gynecol Laparosc 1996; 3 (4 Suppl.): S4-5.

7. Malur S, Possover M, Schneider A. Laparoscopically assisted radical vaginal versus radical abdominal hysterectomy type I in patients with cervical cancer. Surg Endosc 2001; 15: 289-92.

8. Stancu B, Grad NO, Mihaileanu VF, et al. Surgical technique of concomitant laparoscopically assisted vaginal hysterectomy and laparoscopic cholecystectomy. Clujul Med 2017; 90: 348-52.

9. Tapisiz OL, Dogan AR, Kiykac Altinbas S. Vaginal-assisted laparoscopic sacrohysterocervicopexy with retroperitoneal tunneling. Int J Gynaecol Obstet 2018; 140: 118-9.

10. Lanowska M, Brink-Spalink V, Mangler M, et al. Vaginal-assisted laparoscopic radical hysterectomy (VALRH) versus laparoscopic-assisted radical vaginal hysterectomy (LARVH) in the treatment of cervical cancer: surgical results and oncologic outcome. Arch Gynecol Obstet 2014; 289: 1293-300.

11. Gallotta V, Conte C, Federico A, et al. Robotic versus laparoscopic radical hysterectomy in early cervical cancer: a case matched control study. Eur J Surg Oncol 2018; 44: 754-9.

12. Luo C, Liu M, Li X. Efficacy and safety outcomes of robotic radical hysterectomy in Chinese older women with cervical cancer compared with laparoscopic radical hysterectomy. BMC Womens Health 2018; 18: 61.

13. Zhao Y, Hang B, Xiong GW, et al. Laparoscopic radical hysterectomy in early stage cervical cancer: a systematic review and meta-analysis. J Laparoendosc Adv Surg Tech A 2017; 27: 1132-44.

14. Zhang S, Ma L, Meng QW, et al. Comparison of laparoscopic-assisted radical vaginal hysterectomy and abdominal radical hysterectomy in patients with early stage cervical cancer: a retrospective study. Medicine 2017; 96: e8005.

15. Lo CK, Mertz D, Loeb M. Newcastle-Ottawa Scale: comparing reviewers' to authors' assessments. BMC Med Res Methodol 2014; 14: 45.

16. Higgins JP, Thompson SG, Deeks JJ, et al. Measuring inconsistency in meta-analyses. BMJ 2003; 327: 557-60.

17. Choi CH, Lee JW, Lee YY, et al. Comparison of laparoscopic-assisted radical vaginal hysterectomy and laparoscopic radical hysterectomy in the treatment of cervical cancer. Ann Surg Oncol 2012; 19: 3839-48.

18. Gortchev G, Tomov S, Tantchev L, et al. Robot-assisted radical hysterectomy-perioperative and survival outcomes in patients with cervical cancer compared to laparoscopic and open radical surgery. Gynecol Surg 2012; 9: 81-8.

19. Hou CY, Li XL, Jiang F, et al. Comparative evaluation of surgical stress of laparoscopically assisted vaginal radical hysterectomy and lymphadenectomy and laparotomy for early-stage cervical cancer. Oncol Lett 2011; 2: 747-52.
20. Jackson KS, Das N, Naik R, et al. Laparoscopically assisted radical vaginal hysterectomy vs. radical abdominal hysterectomy for cervical cancer: a match controlled study. Gynecol Oncol 2004; 95: 655-61.

21. Morgan DJ, Hunter DC, McCracken G, et al. Is laparoscopically assisted radical vaginal hysterectomy for cervical carcinoma safe? A case control study with follow up. BJOG 2007; 114: 537-42.

22. Naik R, Jackson KS, Lopes A, et al. Laparoscopic assisted radical vaginal hysterectomy versus radical abdominal hysterectomy: a randomised phase II trial: perioperative outcomes and surgicopathological measurements. BJOG 2010; 117: 746-51.

23. Pahisa J, Martinez-Roman S, Torne A, et al. Comparative study of laparoscopically assisted radical vaginal hysterectomy and open Wertheim-Meigs in patients with early-stage cervical cancer: eleven years of experience. Int J Gynecol Cancer 2010; 20: $173-8$.

24. Papacharalabous E, Tailor A, Madhuri T, et al. Early experience of laparoscopically assisted radical vaginal hysterectomy (Coelio-Schauta) versus abdominal radical hysterectomy for early stage cervical cancer. Gynecol Surg 2009; 6: 113-7.

25. Sharma R, Bailey J, Anderson R, et al. Laparoscopically assisted radical vaginal hysterectomy (Coelio-Schauta): a comparison with open Wertheim/Meigs hysterectomy. Int J Gynecol Cancer 2006; 16: 1927-32.

26. Steed H, Rosen B, Murphy J, et al. A comparison of laparascopic-assisted radical vaginal hysterectomy and radical abdominal hysterectomy in the treatment of cervical cancer. Gynecol Oncol 2004; 93: 588-93.

27. Yu JJ, Yang WX, Wang XM. Laparoscopically-assisted radical vaginal hysterectomy with five years follow-up: a case control study. Eur J Gynaecol Oncol 2013; 34: 156-8.

28. Pergialiotis V, Rodolakis A, Christakis D, et al. Laparoscopically assisted vaginal radical hysterectomy: systematic review of the literature. J Minim Invasive Gynecol 2013; 20: 745-53.

29. Querleu D. Laparoscopically assisted radical vaginal hysterectomy. Gynecol Oncol 1993; 51: 248-54.

30. Hertel H, Kohler C, Michels W, et al. Laparoscopic-assisted radical vaginal hysterectomy (LARVH): prospective evaluation of 200 patients with cervical cancer. Gynecol Oncol 2003; 90 : 505-11.

31. Mehra G, Weekes A, Vantrappen P, et al. Laparoscopic assisted radical vaginal hysterectomy for cervical carcinoma: morbidity and long-term follow-up. Eur J Surg Oncol 2010; 36: 304-8.

32. Hagen B, Shepherd JH, Jacobs IJ. Parametrial resection for invasive cervical cancer. Int J Gynecol Cancer 2000; 10: 1-6.

33. Persson J, Reynisson P, Borgfeldt C, et al. Robot assisted laparoscopic radical hysterectomy and pelvic lymphadenectomy with short and long term morbidity data. Gynecol Oncol 2009; 113: $185-90$

Received: 10.12 .2020 , accepted: 19.02 .2021 